\title{
BMJ Open Safety and efficacy of herbal medicine for acute intracerebral hemorrhage (CRRICH): a multicentre randomised controlled trial
} \begin{tabular}{l}
\hline Liling Zeng, ${ }^{1}$ Guanghai Tang, ${ }^{2}$ Jing Wang, ${ }^{3}$ Jianbin Zhong, ${ }^{4}$ Zhangyong Xia, ${ }^{5}$ \\
Jiexia Li, ${ }^{6}$ Guangsheng Chen, ${ }^{7}$ Yongbo Zhang, ${ }^{8}$ Saihua Luo, ${ }^{9}$ Gan Huang, ${ }^{10}$ \\
Qianshan Zhao, ${ }^{11}$ Yue Wan, ${ }^{12}$ Chaojun Chen, ${ }^{13}$ Kaiyun Zhu, ${ }^{14}$ Hanzi Qiao, ${ }^{15}$ \\
Jian Wang, ${ }^{16}$ Tao Huang, ${ }^{15}$ Xian Liu, ${ }^{15}$ Qixin Zhang, ${ }_{15}$ Rongming Lin, ${ }^{17}$ Haijun Li, ${ }^{15}$ \\
Baoying Gong, ${ }^{15}$ Xiuyan Chen, ${ }^{15}$ Yuexiang Zhou, ${ }^{15}$ Zehuai Wen,,${ }^{18}$ Jianwen Guo ${ }^{15}$
\end{tabular}

\section{ABSTRACT}

To cite: Zeng L, Tang G Wang J, et al. Safety and efficacy of herbal medicine for acute intracerebral hemorrhage (CRRICH): a multicentre randomised controlled trial. BMJ Open 2019:9:e024932. doi:10.1136/ bmjopen-2018-024932

- Prepublication history and additional material for this paper are available online. To view these files, please visit the journal online (http://dx.doi. org/10.1136/bmjopen-2018024932).

Received 25 June 2018 Revised 20 March 2019 Accepted 27 March 2019 time window. China.
Check for updates

(c) Author(s) (or their employer(s)) 2019. Re-use permitted under CC BY-NC. No commercial re-use. See rights and permissions. Published by BMJ.

For numbered affiliations see end of article.

Correspondence to Dr Jianwen Guo; jianwen_guo@qq.com
Objective To evaluate the safety and efficacy of removing blood stasis (RBS) herbal medicine for the treatment of acute intracerebral haemorrhage (AICH) within a 6-hour

Study design A randomised, multicentre, double-blind, placebo-controlled study performed in 14 hospitals in

Participants and interventions Patients with $\mathrm{AICH}$ were randomly assigned to receive a placebo, the $\mathrm{ICH}$ 1 (Intracerebral Haemorrhage) formula (eight herbs, including the RBS herbs hirudo and tabanus) or the ICH-2 formula (six herbs without the RBS herbs hirudo and tabanus) within 6 hours of ICH onset.

Outcomes The primary safety outcome was the incidence of haematoma enlargement at 24 hours and at 10 days after treatment. The secondary outcome was the incidence of poor prognosis (mortality or modified Rankin Scale score $\geq 5$ ) assessed at 90 days after symptom onset.

Results A total of 324 subjects were randomised between October 2013 and May 2016: 105 patients received placebo; 108 patients received the ICH-1 formula; and 111 patients received the ICH-2 formula. The incidence of haematoma enlargement at 24 hours was $7.8 \%$ in the placebo group, $12.3 \%$ in the $\mathrm{ICH}-1$ group and $7.5 \%$ in the ICH-2 group; the incidence of haematoma enlargement on day 10 was $1.1 \%$ in the placebo group, $1.1 \%$ in the ICH-1 group, and $3.1 \%$ in the $\mathrm{ICH}-2$ group, with no significant differences among the groups $(\mathrm{P}>0.05)$. The mortality rates were $3.8 \%$ in the placebo group, $2.8 \%$ in the $\mathrm{ICH}-1$ group, and $0.9 \%$ in the $\mathrm{ICH}-2$ group; the incidences of poor prognosis were $7.1 \%$ in the placebo group, $6.0 \%$ in the $\mathrm{ICH}-1$ group and $4.8 \%$ in the $\mathrm{ICH}-2$ group at 3 months, with no significant differences among the groups ( $p>0.05)$. However, the overall frequency of treatment-emergent adverse events in the ICH-1 group (12.1\%) was higher among the three groups $(5.8 \%$ and $2.8 \%$, respectively, $\mathrm{p}<0.05)$. All three cases of serious adverse events were in the ICH-1 group.

Conclusions Ultra-early administration of $\mathrm{ICH}-1$ formula for AICH patients did not exert significant beneficial effects on clinical outcomes but increased the risk of bleeding,

\section{Strengths and limitations of this study}

- The CRRICH trial is the largest multicentre clinical trial to examine the safety and efficacy of two Chinese compound formulas in patients with acute intracerebral haemorrhage (AICH).

- This study highlights the possibility that the early use of removing blood stasis herbal medicine in $\mathrm{AICH}$ could increase the risk of bleeding, which spurs further research on the safety of Chinese medicines.

- There were no available data to elucidate the mechanisms of treatment-emergent adverse events (TEAEs) or serious TEAEs.

which probably resulted from the inclusion of RBS herbal medicines in ICH-1.

Trialregistration number NCT01918722.

\section{INTRODUCTION}

Acute intracerebral haemorrhage (AICH) accounts for approximately $15 \%^{1}$ of all acute strokes; stroke is the third most common cause of death in most Western countries, following coronary heart disease and cancer. ${ }^{23}$ AICH has been the leading cause of death in China in recent years ${ }^{4}$ and is responsible for approximately one-third of the total number of deaths from stroke worldwide. ${ }^{5}$

Available specific medical and surgical treatments do not improve prognosis substantially, ${ }^{6} 7$ and AICH still accounts for a higher proportion of stroke in Chinese people than in white populations. ${ }^{8}$ Therefore, traditional Chinese medicine (TCM) physicians in China widely use removing blood stasis (RBS) herbs for the treatment of AICH. Meta-analysis showed that RBS herbal therapy for AICH could improve the neurological function 
deficit, reduce the volume of haematoma and perihaematomal oedema and lower the mortality rate and dependency. ${ }^{9}$ It is possible that RBS herbal medicine promotes haematoma absorption and improves the recovery of neurological impairment ${ }^{10}{ }^{11}$; RBS herbal medicine has already been included by guidelines for the management of spontaneous ICH. However, sufficient evidence of longterm benefits and risks of superacute stage is lacking. RBS herbals, such as Hirudo nipponica Whitman, which acts as a thrombin inhibitor with anticoagulant pharmacological effects, may cause rebleeding risk in clinical practice. ${ }^{12}$ A total of $90 \%$ of haematoma growth, which plays an important role in prognosis, occurs within a 6-hour time window. ${ }^{13}$ Therefore, a confirmation of the safety and efficacy of RBS herbal medicines in the treatment of earlystage AICH is urgently needed.

We performed a retrospective study that demonstrated that RBS herbal medicine for patients with AICH was safe and effective. ${ }^{14}$ However, little additional evidence of the safety and efficacy of this treatment exists. We designed a multicentre, three-group, prospective, randomised, double-blinded and placebo-controlled clinical trial on haematoma enlargement in patients with AICH treated with RBS herbal medicine within a 6-hour time window from symptom onset (CRRICH: Clinical re-evaluation of removing blood stasis therapy in treating acute intracerebral hemorrhage) to evaluate its safety and efficacy in patients with AICH and to provide a high level of evidence for clinical practice.

\section{METHODS}

\section{Study design}

The CRRICH trial was a multicentre, randomised, double-blinded, placebo-controlled, parallel-group study to assess the safety and efficacy of RBS herbal medicine in patients with AICH. This trial is registered at clinicaltrials. gov (NCT01918722), and the research design proposal has been published. ${ }^{15}$

\section{Participants}

Patients with a Glasgow Coma Scale (GCS) score $\geq 6$ were enrolled if they were $\geq 18$ years of age and had a clinical diagnosis of ICH confirmed with CT within 6 hours of onset. All participants signed an informed consent form. If the participants did not have the capacity to sign the informed consent for serious condition or illiteracy, the researcher will explain the informed consent to the patients or their authorised immediate family. Weighing the pros and cons of both sides, their immediate family will decide whether to sign the informed consent on behalf of the patient. Patients who suffered secondary ICH resulting from trauma, brain tumour, blood diseases, arteriovenous malformation or aneurysm were excluded. A detailed patient history was recorded. Online supplementary table S1 provides a full list of study inclusion and exclusion criteria during the screening period.

\section{Randomisation and blinding}

Patients were randomised to receive placebo, ICH-1 or ICH-2 in a 1:1:1 ratio with a stratification and block size of 6 using the PROC PLAN process in SAS software V.9.13. Stratified block and randomisation were concealed using sequentially numbered opaque envelopes. Persons involved in the study, including the investigators, patients and data analysts, were blinded to treatment assignment during double-blinded treatment until the end of follow-up. Only the data administrators were permitted access to unblinded data.

\section{Procedures}

\section{Enrolment, intervention and follow-up}

Patients were recruited from October 2013 through May 2016 at 14 hospitals in China (see online supplementary table S2). The study consisted of a screening and randomisation period ( $\leq 6$ hours poststroke), a 10-day doubleblinded treatment period and a 3-month follow-up period (see online supplementary figure S1). Participants were randomly assigned to one of three treatment groups at a ratio of 1:1:1 during the double-blinded treatment (shown in the online supplementary table S3 and S4): (1) The ICH-1 group received the ICH-1 formula, which consisted of eight herbs including RBS medicines; (2) The ICH-2 group received the ICH-2 formula, which consisted of six herbal medicines without RBS herbs $(H$. nipponica Whitman and Tabanus bivittatus Matsumura) in the same doses as those in the ICH-1 group, with the remaining dose filled with a placebo; and (3) The placebo group received a placebo that included dextrin and farina. Each unit of TCM or placebo was dissolved in $200 \mathrm{~mL}$ of boiling water. Each patient received $200 \mathrm{~mL}$ orally or through a nasogastric tube twice daily for 10 days (see online supplementary table S3).

Patients were followed up for 3 months after the 10-day double-blinded treatment period. Patients were hospitalised in stroke units and received standard stroke care in accordance with local guidelines, which included general supportive care, treatment of acute complications and rehabilitation. Astringents and other RBS drugs were prohibited. Visits were scheduled at baseline, 24 hours poststroke and at the end of the treatment period, with a single visit scheduled 3 months poststroke (see online supplementary figure S1). Adverse events (AEs), treatment compliance and concomitant medication use were reported during each visit. Participants were randomly assigned to one of three treatment groups at a ratio of 1:1:1 during double-blinded treatment.

\section{Primary and secondary outcomes}

The incidence of haematoma enlargement, which was defined as the percentage of participants who experienced haematoma enlargement, was the primary outcome of the study. Haematoma enlargement was operationally defined as an increase in haematoma volume of $>33 \%$ or $12.5 \mathrm{~mL}$, as measured on image analysis on the 24-hour CT 
compared with the baseline CT scan. Haematoma volume was measured using the $\mathrm{ABC} / 2$ Coniglobus formula ${ }^{16}$ at 24 hours after onset. The same measurement was performed on days 10-14 as a primary outcome measure. National Institutes of Health Stroke Scale (NIHSS) scores range from 0 (normal) to 42 (coma with quadriplegia); and modified Rankin Scale (mRS) scores range from 0 (no symptoms or disability) to 6 (death). Both of them can be used to evaluate the clinical outcomes.The following secondary end points were assessed: change in NIHSS score at 3 months, mortality at 3 months and poor prognosis rate, which was defined as the proportion of patients who died or were severely disabled based on an mRS score $\geq 5$ at 3 months. ${ }^{17}$ Safety data focused on treatment-emergent AEs (TEAEs). TEAEs were defined as AEs that first occurred or worsened (increased in severity) after the first dose of study drug. The patient spontaneously reported TEAEs in response to an openended question from the contact person at each visit. A worsened patient condition, prolonged patient hospitalisation and life-threatening TEAEs in patients were reported as serious AEs (TEsAEs). Abnormal laboratory values that constituted a $\mathrm{SAE}$ or led to the discontinuation of experimental drugs were also reported as TEsAEs. Online supplementary table S5 provides a full list of all primary, secondary and safety end points.

\section{Statistical analyses}

All reported efficacy analyses were predefined and based on the full analysis set (FAS) (patients who were randomised and received any study medication) according to the intent-to-treat (ITT) principle. Supportive analyses were performed in a predefined per-protocol population set (PPS), which included FAS patients who did not have one or more key protocol deviations, including violations of inclusion and exclusion criteria. The last observation for surviving patients with missing outcome data was carried forward. Patients who had died by the 90-day follow-up were assigned a Barthel index score of 0 and an mRS score of 6 , and the last recorded NIHSS score was carried forward. The incidence of haematoma enlargement at 24 hours (primary end point), the rate of poor prognosis and the incidence of total TEAEs among the three groups were analysed using the $\chi^{2}$ test. The incidence of haematoma enlargement on days 10-14 (primary end point) and mortality among the three groups were compared using Fisher's exact test. The mean differences in NIHSS score among groups were analysed using one-way analysis of variance. Demographic characteristics and serious TEAEs were summarised using descriptive statistics.

Power and sample size were determined using relative haematoma volume at 24 hours as the primary outcome variable. A total of 285 participants were required at the final follow-up to provide $90 \%$ power with a two-sided alpha level at 0.05 to test the primary efficacy, which was calculated using the PASS (Power Analysis and Sample Size) software programme (licensed by NCSS, LLC). A total of 360 participants were needed for primary outcome randomisation assuming a dropout rate of $20 \%$, with 120 patients per treatment arm. The statistical package STATA (V.13.1) was used for all statistical analyses.

\section{Patient and public involvement statement}

While we put forward the research question and outcome measures, the patients' priorities, experience and preferences were fully considered by a survey. Patients were involved in the recruitment to and conduct of the study. The results would be disseminated to study participants by posting a letter. The CRRICH trial was a randomised controlled trial, and the patients themselves had fully assessed the burden of the intervention. Patient advisors should also be thanked in the contributorship statement/ acknowledgements.

\section{Funding and ethical statements}

The State Administration of Traditional Chinese Medicine of the PRC (SATCM) awarded Guangdong Provincial Hospital of TCM (GPHTCM) SATCM grant no: ZDJX2012074 and provided input during the study period. This report was an independent study supported by SATCM and performed by the GPHTCM in a SATCMGPHTCM partnership. The viewpoints stated in this manuscript are those of the authors and may not represent the opinions of SATCM, GPHTCM or the Public Health Bureau. The SATCM and GPHTCM approved the decision to submit this paper for publication. Dr Jianwen Guo had full access to all study data and had final responsibility for the decision to submit for publication.

Each respective institutional review board or ethics committee approved the study protocol, which followed the established Good Clinical Practice guidelines. All patients provided written informed consent to participate in the study. An independent contract research organisation, S\&R (Department of Science and Research of Guangdong Provincial Hospital of Chinese medicine), managed the administration, coordination and monitoring of the study, including researcher training, study inspection, data management and statistical analyses, with oversight by the SATCM.

\section{RESULTS}

\section{Study population}

Patient recruitment began in October 2013, and the study ended in May 2016. A total of 5589 individuals with $\mathrm{ICH}$ were screened (figure 1). The most frequent reasons for exclusion in descending order were time window $>6$ hours, refusal to participate, secondary ICH (ie, not AICH) and other reasons. A total of 319 of the 324 randomised patients (105 placebo, 108 ICH-1, 111 ICH-2) received the assigned treatment following the elimination of five patients (one patient for missing the time window, one patient for kidney failure, one patient for secondary ICH, one patient for surgery within 24 hours and one patient withdrew consent). Therefore, only the 319 participants who underwent randomisation 


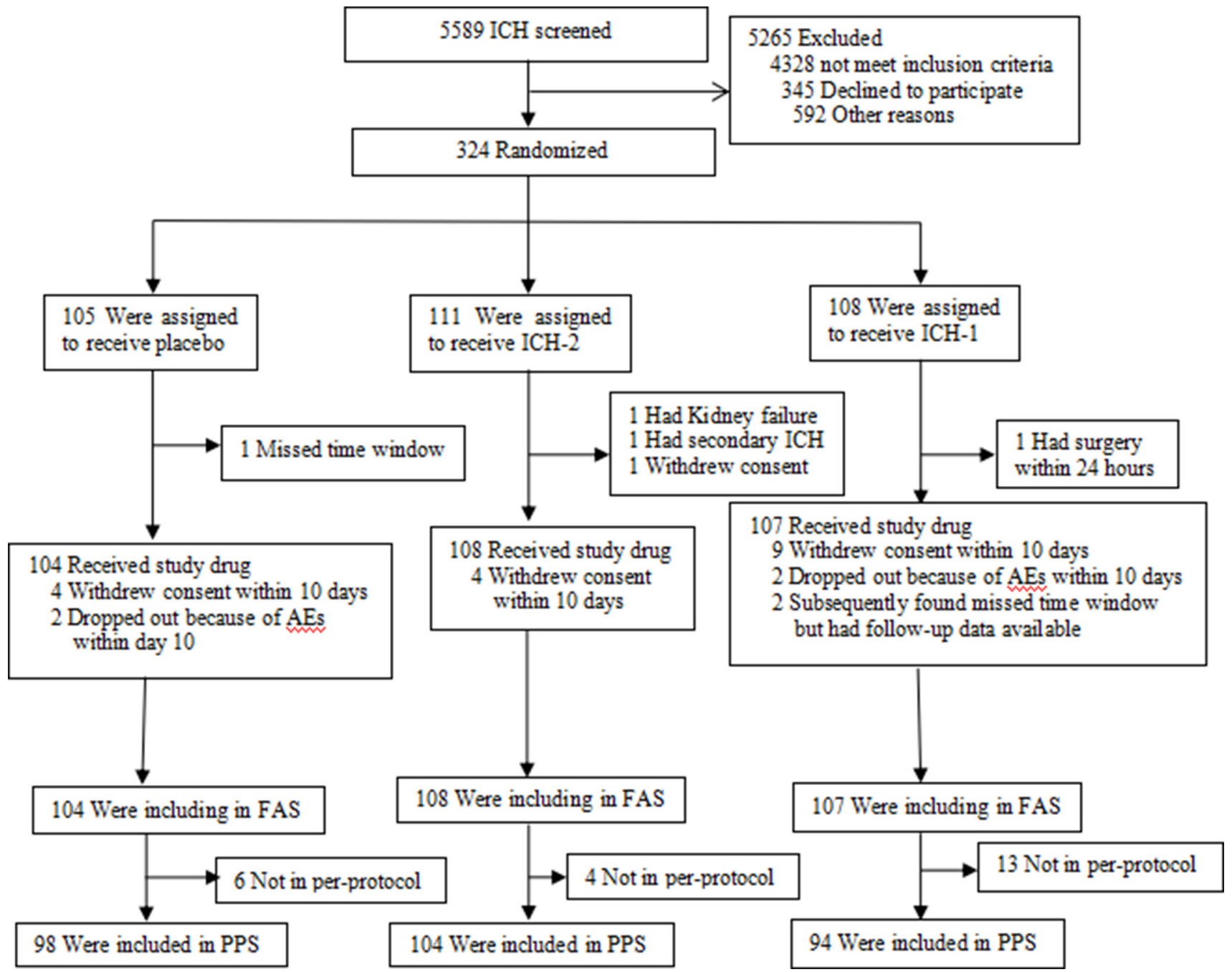

Figure 1 Enrolment and follow-up. AEs, adverse events; FAS, full analysis set; PPS, per-protocol population set; RBS, removing blood stasis; $\mathrm{ICH}$ denotes intracerebral haemorrhage; $\mathrm{ICH}-1$ denotes herbal medicine with RBS herbals hirudo and tabanus (eight herbals); ICH-2 denotes herbal medicine without RBS herbals hirudo or tabanus (six herbals).

and received study medication were included in the FAS and safety analysis set based on ITT. Twenty-three patients in the FAS did not completely adhere to the protocol and dropped out during the treatment period. A total of 296 patients were included in the PPS (see figure 1 for patient flow diagram). The mean dropout rate was $<8 \%$. Table 1 presents the demographics and baseline characteristics of the FAS, which were well balanced with no significant between-group differences. The mean age was $62.5 \pm 12.7$ years (mean $\pm \mathrm{SD}, \mathrm{n}=319$ ); $65.5 \%$ of the patients were men, and $98.7 \%$ were ethnic Han. The median GCS score was 15 (range, 3 to 15), and the mean NIHSS score was 8 (range, 0 to 47). The deep grey matter was involved in $93.4 \%$ of cases, and the lobar regions were involved in $6.6 \%$ of cases. The baseline characteristics of the three treatment groups were similar. The mean volume of the ICH at baseline for all patients was $11.0 \mathrm{~mL}$ (range, 0.3 to $86.7 \mathrm{~mL}$ ), which was similar in the three groups (table 1). The mean time from onset of symptoms to admission was $3.36 \pm 1.49$ hours, and the mean time from onset of symptoms to treatment was $4.10 \pm 1.38$ hours. Fourteen per cent of patients were treated within 3 hours after symptom onset. Two patients were treated outside of the 6-hour time window. The timing of the treatment was similar in the three groups (table 1 ).

\section{Primary outcome (radiographic outcomes)}

At 24 hours, a total of 319 baseline CT scans and 315 CT scans were available for analysis. The incidence of haematoma enlargement were $7.8 \%$ in the placebo group, $12.3 \%$ in the ICH-1 group and $7.5 \%$ in the ICH-2 group. There was no significant difference in the incidence of haematoma enlargement at 24 hours among the three groups $(p=0.409)$. The incidence of haematoma enlargement on days $10-14$ was $1.1 \%$ in the placebo group, $3.1 \%$ in the ICH-2 group and $1.1 \%$ in the ICH-1 group. There was also no significant difference among the three groups in the incidence of haematoma enlargement on days 10-14 $(\mathrm{p}=0.625)$ (table 2).

\section{Secondary outcomes (clinical outcomes)}

Mortality at 3 months was approximately $2.6 \%$ in the three groups (table 2). Poor prognosis (ie, the proportion 
Table 1 Baseline characteristics and treatment timing

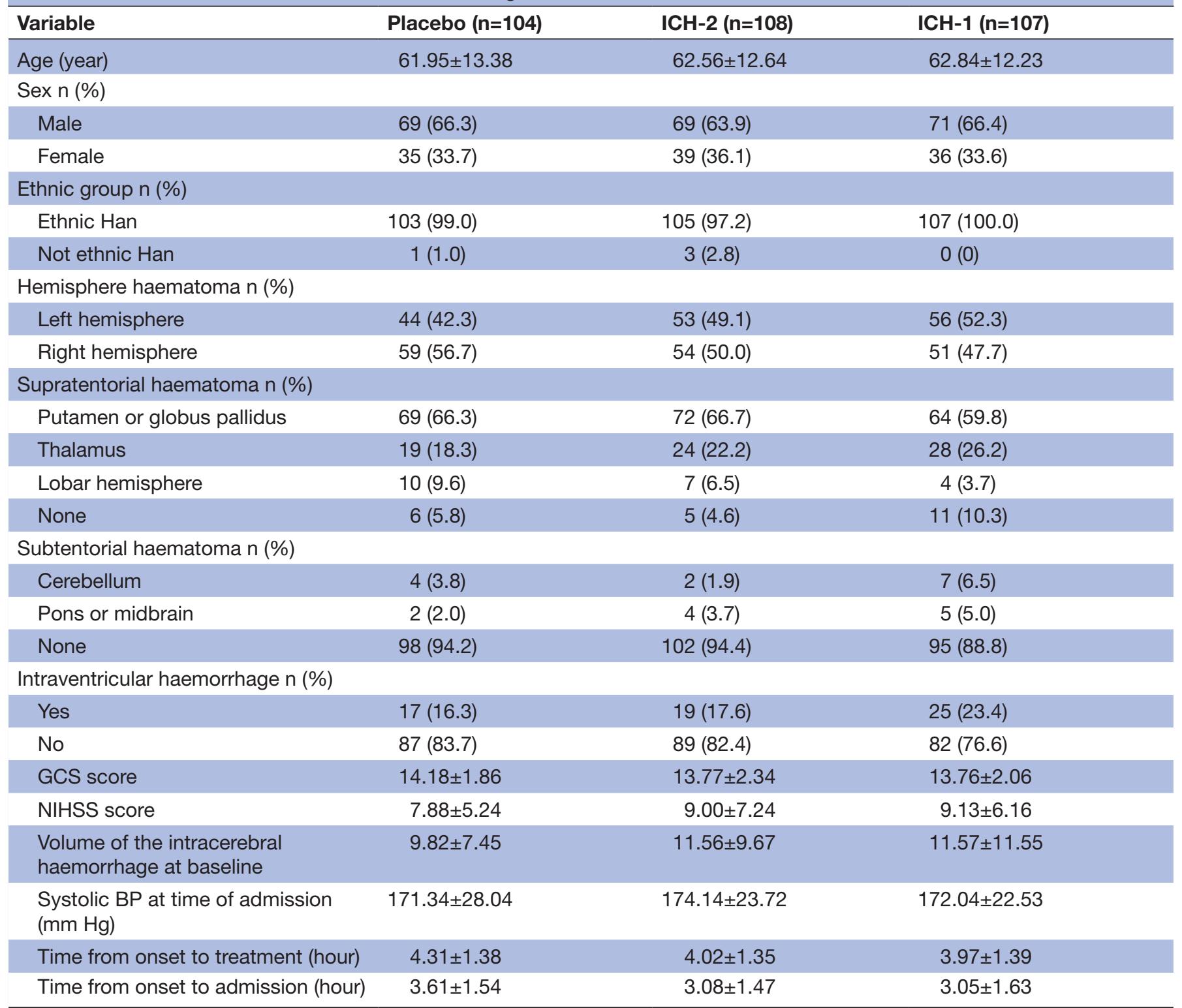

ICH denotes intracerebral haemorrhage; GCS, Glasgow Coma Scale (scores range from 15 [normal] to 3 [deep coma]); NIHSS, National Institutes of Health Stroke Scale (scores range from 0 (normal) to 42 (coma with quadriplegia)); BP, blood pressure.

of patients who died or were severely disabled, $\mathrm{mRS} \geq 5$ ) did not differ significantly among the three groups. There are less more patients with poor prognosis after ICH-2 treatment, but no significant difference compared with placebo treatment (table 2). The distributions of outcomes on the mRS (figure 2) were similar among the three groups. The differences in the NIHSS scores at 3 months among the three groups were not significant (table 2).

\section{Adverse events}

There were altogether 35 AEs reported by investigators, of which 22 AEs may have been TEAEs. All the TEAEs occurred during the double-blinded treatment period (within 2 weeks). TEAEs occurred in 13 (12.1\%) ICH-1treated patients, $3(2.8 \%)$ ICH-2-treated patients and 6
$(5.8 \%)$ placebo-treated patients. The patterns of TEAEs were similar in the three groups, but the overall frequencies of TEAEs among the three groups were significantly different $(p=0.022)$, as shown in table 2 . And the paired comparisons showed that the test for difference in the overall TEAEs rate between group ICH-1 and group ICH-2 yields a $\mathrm{p}$ value of 0.029 . Table 3 shows the TEAEs that occurred during the study. The most frequent event was diarrhoea, and its incidence was similar in the three treatment groups. Treatment was discontinued because of TEsAEs in three (1.9\%) ICH-1-treated patients. The three cases with TEsAEs (two cases of gastrointestinal bleeding, one case of cerebral hernia) reported by investigators occurred in ICH-1-treated patients. There was no consistent pattern to this difference, and all serious 


$\begin{array}{llll}\text { Placebo } & \mathrm{ICH}-2 & \mathrm{ICH}-1 & \text { P value }\end{array}$

\begin{tabular}{|c|c|c|c|c|}
\hline \multicolumn{5}{|c|}{ Primary outcome: the incidence of haematoma enlargement at 24 hours and at day 14} \\
\hline At 24 hours & $8 / 104(7.8)$ & $8 / 108(7.5)$ & 13/107 (12.3) & 0.409 \\
\hline On days 10-14 & $1 / 92(1.1)$ & $3 / 97(3.1)$ & $1 / 91(1.1)$ & 0.625 \\
\hline At baseline & $9.82 \pm 7.45$ & $11.56 \pm 9.67$ & $11.57 \pm 11.55$ & 0.284 \\
\hline At 24 hours & $9.71 \pm 6.94$ & $11.97 \pm 10.02$ & $14.44 \pm 19.33$ & 0.313 \\
\hline $\begin{array}{l}\text { Millilitres of increase from } \\
\text { baseline }\end{array}$ & $-0.13 \pm 1.41$ & $0.22 \pm 2.24$ & $3.13 \pm 16.10$ & 0.168 \\
\hline \multicolumn{5}{|l|}{ Secondary outcomes: } \\
\hline NIHSS* at 3 months & $3.58 \pm 5.32$ & $3.58 \pm 5.32$ & $3.58 \pm 5.32$ & 0.475 \\
\hline
\end{tabular}

The values are expressed as $\mathrm{n} / \mathrm{N}(\%)$ within group or the means $\pm \mathrm{SD}$;

*Denotes the number of patients at 3 months: 104 in the placebo group, 107 in the ICH-2 group and 105 in the ICH-1 group.

ICH denotes intracerebral haemorrhage; NIHSS, National Institutes of Health Stroke Scale; TEAEs, treatment-emergent adverse events.

TEAEs occurred within the 10-day drug treatment period. There were no statistically significant differences in the incidence or type of serious TEAEs leading to death among groups (data not shown). There was no relationship between TEAEs, serious TEAEs or mortality and poor outcome (data not shown).

\section{DISCUSSION}

ICH-1 administration within 6 hours after the symptom onset of ICH did not significantly reduce haematoma growth and failed to improve survival or functional outcome at 90 days. Conversely, compared with the ICH-2,

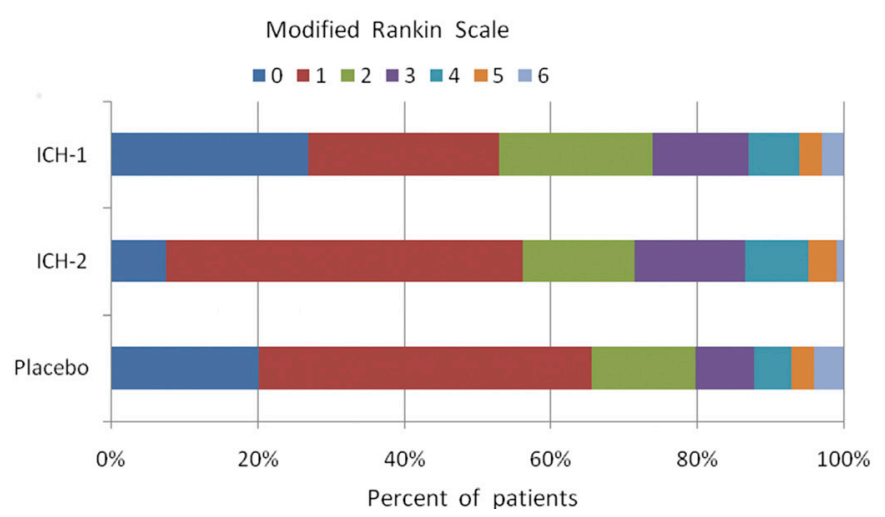

Figure 2 Clinical outcome at 90 days according to the modified Rankin Scale. The modified Rankin Scale evaluates global disability and handicap. Scores range from 0 (no symptoms or disability) to 6 (death). There were no significant differences among the three groups. the incidence of TEAEs showed an increasing trend in the ICH-1 group ( $p=0.029$, ICH-1 vs ICH-2). Significant increases were also demonstrated in total TEAE frequency and in serious TEAEs in the ICH-1 group. Three serious bleeding events occurred in the ICH-1 group (ICH-1, with two RBS herbals), including two cases of gastrointestinal bleeding and one case of intracerebral rebleeding. All three cases were fatal serious TEAEs (table 2). These data suggest that RBS treatment for ICH patients within 6 hours of symptom onset is a safety concern. However, no data were available to suggest a mechanism for this effect. The possible rebreeding mechanism is that RBS

\begin{tabular}{|c|c|c|c|}
\hline & Placebo & $\mathrm{ICH}-2$ & $\mathrm{ICH}-1$ \\
\hline Vomiting & $1 / 104(1.0)$ & $0 / 108(0.0)$ & 2/107 (1.9) \\
\hline $\begin{array}{l}\text { Gastrointestinal } \\
\text { bleeding* }\end{array}$ & $0 / 104(0.0)$ & $0 / 108(0.0)$ & $2 / 107$ (1.9) \\
\hline Stomachache & $0 / 104(0.0)$ & $0 / 108(0.0)$ & $1 / 107(0.9)$ \\
\hline Chest congestion & $1 / 104(1.0)$ & $0 / 108(0.0)$ & $0 / 107(0.0)$ \\
\hline Cerebral hernia* & $0 / 104(0.0)$ & $0 / 108(0.0)$ & $1 / 107(0.9)$ \\
\hline
\end{tabular}

The values are expressed as $\mathrm{n} / \mathrm{N}(\%)$ within group. All adverse events are listed in order of frequency from the overall study period (90 days); adverse events were collected from investigator reports. The principal investigator at each site determined whether an adverse event or serious adverse event was related to the intervention.

*Indicates TEAEs.

TEAEs, treatment-emergent adverse events . 
medicine, such as H. nipponica Whitman, which has anticoagulant pharmacological effects, could increase bleeding risk according to the previous literature. ${ }^{12}$

As can be seen from the table 2, ICH-2 treatment was least vulnerable to poor prognosis and TEAEs. Therefore, ICH-2 formula would have the potential to be an effective herbal drug for ICH patients of superacute stage.

This result contrasts with the clinical benefit demonstrated in a previous meta-analysis, ${ }^{9}$ which included nine randomised controlled clinical trials with 798 individuals, demonstrating that RBS therapy for acute ICH reduced brain haematoma and cerebral oedema volumes, improved the neural function and reduced the mortality and disability rates with fewer TEAEs. The present trial revealed significant heterogeneity compared with the studies included in the meta-analysis, possibly because of differences in the intervention time window. This research is the first prospective, multicentre, randomised, doubleblind, placebo-controlled study to assess the efficacy and safety of ICH-1 in patients with AICH within a 6-hour time window from symptom onset, unlike prior studies that examined these effects within a 24-hour or later intervention time window. Earlier RBS administration increased the risk of bleeding events (eg, gastrointestinal bleeding or intracerebral rebleeding), which counteracted the benefit to some extent.

The primary outcome of the incidence of haematoma enlargement, which is an independent prognostic determinant of mortality and poor prognosis, ${ }^{18}$ was also used to assess safety. The incidence of haematoma enlargement at 24 hours in the three groups in the CRRICH trial (placebo, ICH-2, and ICH-1) were $7.8 \%, 7.5 \%$ and $12.3 \%$, respectively, and they were not significantly different (table 2). The mean incidence of haematoma enlargement was only $9.2 \%$, which was far lower than the mean level of $18 \%-30 \% .^{18}$ The main reasons for lower haematoma expansion incidence may include the following: (1) the investigators' inclination toward surgery in patients with AICH with large haemorrhage volumes within 24 hours of onset, which resulted in the exclusion of patients susceptible to rebleed in the study; and (2) patients with terrible and unstable conditions who were prone to haematoma expansion were excluded by investigators privately for fear that deterioration risk would increase during study.

Haematoma growth is an independent determinant of death and disability. ${ }^{19}$ Therefore, haematoma expansion may be an attractive therapeutic target. ${ }^{20}{ }^{21}$ However, no proven specific therapies or treatments exist to prevent haematoma expansion or improve outcomes after ICH. Sufficient evidence-based medical research to prove that minitraumatic surgeries and the present available medicines provide ideal treatment is lacking. ${ }^{67}$

Herbal medicine in China has been used to treat ICH for at least 2000 years, and RBS herbal medicines are listed in the 2010 Chinese Pharmacopoeia. ${ }^{22} \mathrm{Dr} \mathrm{Si}$ reported the earliest available literature on AICH with RBS herbal medicine therapy in $1981 .^{23}$ Another
RBS medicine, Naoxuekang capsule, was approved as routine treatment for patients with $\mathrm{AICH}$ by the Chinese State Food and Drug Administration ${ }^{22}$ (Naoxuekang capsule, register no. Z10960009). RBS herbal medicines have been studied in patients with AICH in more than 200 clinical studies over the past 30 years, and they have gradually come to be considered a regular therapy. ${ }^{24}$ AICH management guideline in China cited this treatment. ${ }^{25}$ However, safety evidence in the early stage of AICH is lacking. Some studies demonstrated that $30 \%$ of ICH patients suffered continued bleeding within 6 hours of onset. ${ }^{26}$ Some research has provided low-level evidence of the safety of RBS herbal medicine for superacute cerebral haemorrhage, but whether RBS herbal medicine administration in an early-stage induced haematoma growth was not known because RBS herbal medicine exhibits an anticoagulation effect. ${ }^{27}$ Marketed RBS drugs that are clinically recognised and widely used did not illustrate a specific application time window. Early administration of RBS drugs may cause haematoma expansion and increase the risk of death and serious disability. The CRRICH trial was a prospective, double-blinded, multicentre clinical trial that demonstrated that early administration of RBS drugs did not increase haematoma growth but significantly increased TEAEs.

The CRRICH trial is the largest clinical trial to investigate the safety and efficacy of a Chinese compound formula in patients with AICH. Three other influential RCT trials of medical treatments with drugs, including FAST, INTERACT2 and CHANT, that exhibit mechanisms very different from those of RBS used similar methods to evaluate the safety and efficacy and found no significant benefit. $^{1728} 29$

Data from the clinical study suggested that the administration of RBS herbal medicine should be avoided in patients with AICH during the initial 6-hour time window from stroke onset to avoid the increased risk of bleeding. Further clinical studies should be performed to confirm the safety and efficacy of these agents beyond the 6 hours of ICH onset. This research suggests that the early administration of RBS herbal medicine in ICH patients increases the risk of bleeding, such as gastrointestinal bleeding, and haematoma growth. Some Chinese patent drugs (Naoxuekang capsule [Z10960009], Naoxuekang pill [Z20050312] or Naoxuekang drop pills [Z10980039]), which primarily consist of Hirudo nipponica Whitman, should be re-evaluated and used under supervision. The relevant manufacturers should revise the drug instructions and add a warning, 'Do not use for superacute cerebral haemorrhage within 6 hour from onset', if necessary.

This study had some limitations. First, there were no available data to elucidate the mechanism of TEAEs or serious TEAEs. Second, there was a significant difference in the number of patients among research centres. Third, the investigators preferred to study patients with minor haematoma volume because they were afraid of the rebleed risk from the herbal medicine. 


\section{Summary}

This study demonstrates that ultra-early administration, namely within 6 hours of ICH onset, of RBS herbal medicine for patients with AICH increased the risk of bleeding, such as gastrointestinal bleeding, and haematoma growth. This study also shows that RBS herbal medicine did not exert significantly beneficial effects on clinical outcomes in hypertensive ICH patients in the ultra-early stage. These results support the idea that the ultra-early administration of RBS herbal medicine is unsafe and has a low efficacy. Future high-quality research should further assess the efficacy and safety of RBS herbal medicine for intracerebral haemorrhage beyond the 6-hour time window.

\section{Author affiliations}

${ }^{1}$ The Second Clinical Medical College, Guangzhou University of Chinese Medicine, Guangdong Provincial Hospital of Chinese Medicine, Guangzhou, China

${ }^{2}$ Neurology, Shenyang No. 2 hospital of traditional Chinese Medicine, Shenyang, China

${ }^{3}$ Neurology, Shenzhen Longhua New District Center Hospital, Shenzhen, China

${ }^{4}$ Neurology, Boji-affiliated Hospital of Sun Yat-sen University, Guangzhou, China

${ }^{5}$ Neurology, Liaocheng People's Hospital, Liaocheng Clinical School of Taishan Medical University, Liaocheng, China

${ }^{6}$ Neurology, Guangzhou Conghua district Hospital of Traditional Chinese Medicine, Guangzhou, China

${ }^{7}$ Neurology, Boluo County People's Hospital, Huizhou, China

${ }^{8}$ Neurology, Shouguang City People's Hospital, Shouguang, China

${ }^{9}$ Neurology, Lianjiang People's Hospital, Lianjiang, China

${ }^{10}$ Neurology, Yangjiang Hospital of Traditional Chinese Medicine, Yangjiang, China

${ }^{11}$ Neurology, Jiangmen Wuyi Hospital of Chinese Medicine, Jiangmen, China

${ }^{12}$ Neurology, Zhongshan Hospital of Hubei Province, Wuhan, China

${ }^{13}$ Neurology, Guangzhou Hospital of Integrated Traditional and West Medicine, Guangzhou, China

${ }^{14}$ Neurology, Panyu district Hospital of Chinese Medicine, Guangzhou, China

${ }^{15}$ Neurology, Guangdong Provincial Hospital of Chinese Medicine, Guangzhou, China

${ }^{16}$ Neurology, First Affiliated Hospital to Changchun University of Chinese Medicine,

Changchun, Jilin, China

${ }^{17}$ Neurology, Guangdong Second Hospital of Traditional Chinese Medicine, Guangzhou, China

${ }^{18}$ Key Unit of Methodology in Clinical Research, Guangdong Provincial Hospital of Chinese Medicine, Guangzhou, China

Acknowledgements We thank the patients and families who volunteered for this study, Zehuai Wen and Ouyang Wen for their assistance with data summarisation and Yubo Lv for his guidance. The CRRICH trial was supported by an SATCM Grant (No: ZDJX2012074) awarded to Dr Jianwen Guo from the State Administration of Traditional Chinese Medicine of the PRC (SATCM). We also thank Guangdong Provincial Key Laboratory of Research on Emergency in TCM(2017B030314176).

Contributors JG, LZ and JW organised the trial hypotheses, designed the trial and provided guidance about the data analysis and interpretation/presentation of the data. JG is the subject primarily responsible of the clinical trial (CRRICH trial) and provided a critical review of the manuscript. GT designed the patient's medical and surgery treatment protocol. LZ drafted most sections of the manuscript. JL, GC, YZ(Yongbo Zhang), SL, GH, QZ,GT and YW were involved in the design of the study and contributed to writing and revising the manuscript. GT, JZ, ZX, RL, CC, KZ, and $\mathrm{HQ}$ organised and managed the trial including trial start-up, data collection, quality assurance and trial close-out. XL provided the region of interest calculations for all volumetric measurement results. JW and TH provided an independent review and adjudication of all safety events. QZ, HL, BG, XC, YZ(Yuexiang Zhou) and ZW were involved in the statistical analysis and data interpretation and contributed to the development of and revisions to the manuscript. LZ and GT have equal contribution for this manuscript.

Funding The State Administration of Traditional Chinese Medicine of the PRC (SATCM) has allocated to Guangdong Provincial Hospital of TCM (GPHTCM): SATCM grant no: ZDJX2012074, JDZX2015048 and a Project of the Department of Science and Technology of Guangdong Province, grant no. 2014A020221074. JG is the subject primarily responsible. This report is an independent study supported by the SATCM and conducted by the GPHTCM in the name of the SATCM-GPHTCM partnership.

Disclaimer The viewpoints stated in this manuscript are those of the authors and may not be those of the SATCM, GPHTH or the Public Health Bureau.

Competing interests None declared.

Patient consent for publication Obtained.

Ethics approval B2013-085-01; the CRRICH trial was approved by the institutional review boards at all participating centers (specified in the text).

Provenance and peer review Not commissioned; externally peer reviewed. Data sharing statement No additional unpublished data are available.

Open access This is an open access article distributed in accordance with the Creative Commons Attribution Non Commercial (CC BY-NC 4.0) license, which permits others to distribute, remix, adapt, build upon this work non-commercially, and license their derivative works on different terms, provided the original work is properly cited, appropriate credit is given, any changes made indicated, and the use is non-commercial. See: http://creativecommons.org/licenses/by-nc/4.0/.

\section{REFERENCES}

1. Qureshi Al, Mendelow AD, Hanley DF. Intracerebral haemorrhage. Lancet 2009;373:1632-44.

2. Ng M, Fleming $\mathrm{T}$, Robinson $\mathrm{M}$, et al. Global, regional, and national prevalence of overweight and obesity in children and adults during 1980-2013: a systematic analysis for the Global Burden of Disease Study 2013. Lancet 2014;384:766-81.

3. Feigin VL, Krishnamurthi RV, Parmar P, et al. Update on the Global Burden of Ischemic and Hemorrhagic Stroke in 1990-2013: The GBD 2013 Study. Neuroepidemiology 2015;45:161-76.

4. Liu L, Wang D, Wong KS, et al. Stroke and stroke care in China: huge burden, significant workload, and a national priority. Stroke 2011;42:3651-4.

5. van Asch CJ, Luitse MJ, Rinkel GJ, et al. Incidence, case fatality, and functional outcome of intracerebral haemorrhage over time, according to age, sex, and ethnic origin: a systematic review and meta-analysis. Lancet Neurol 2010;9:167-76.

6. Hemphill JC, Greenberg SM, Anderson CS, et al. Guidelines for the Management of Spontaneous Intracerebral Hemorrhage: A Guideline for Healthcare Professionals From the American Heart Association/ American Stroke Association. Stroke 2015;46:2032-60.

7. Rabinstein AA. Intracerebral haemorrhage: no good treatment but treatment helps. Lancet 2017;389:575-6.

8. Tsai CF, Thomas B, Sudlow CL. Epidemiology of stroke and its subtypes in Chinese vs white populations: a systematic review. Neurology 2013;81:264-72.

9. Li HQ, Wei JJ, Xia W, et al. Promoting blood circulation for removing blood stasis therapy for acute intracerebral hemorrhage: a systematic review and meta-analysis. Acta Pharmacol Sin 2015;36:659-75.

10. Wang $Y Q$, Shi $Q$, Wang WP. Clinical study of xuefuzhuyu decoction on hypertensive intracerebral hemorrhage. J Emerg Tradit Chin Med 2013;22. 1686-7, 1689. Chinese.

11. Zhang ZZ, Zhang BH, Chen M. The study of Danshen Injection for prevention and treatment of brain edema in acute intracerebral hemorrhage. Zhejiang J Integr Tradit Chin West Med 2003;13:355-7.

12. Monreal M, Costa J, Salva P. Pharmacological properties of hirudin and its derivatives. Potential clinical advantages over heparin. Drugs Aging 1996;8:171-82.

13. Brott T, Broderick J, Kothari R, et al. Early hemorrhage growth in patients with intracerebral hemorrhage. Stroke 1997;28:1-5.

14. Xu Y, Guo J, Liu X, et al. Can Herbal Medicine Cause Hematoma Enlargement of Hypertensive Intracerebral Hemorrhage within $24 \mathrm{hrs}$ Time Window? A Retrospective Study of 256 Cases from a Single Center in China. Evid Based Complement Alternat Med 2015;2015:1-8.

15. Zeng L, Guo J, Wang J, et al. Clinical re-evaluation of removing blood stasis therapy in treating acute intracerebral hemorrhage safety and efficacy: a protocol for a randomized, controlled, multicenter study (CRRICH Trial). Springerplus 2016;5:1466.

16. Kothari $\mathrm{RU}$, Brott T, Broderick JP, et al. The ABCs of measuring intracerebral hemorrhage volumes. Stroke 1996;27:1304-5.

17. Mayer SA, Brun NC, Begtrup K, et al. Efficacy and safety of recombinant activated factor VII for acute intracerebral hemorrhage. N Engl J Med 2008;358:2127-37. 
18. Demchuk AM, Dowlatshahi D, Rodriguez-Luna D, et al. Prediction of haematoma growth and outcome in patients with intracerebral haemorrhage using the CT-angiography spot sign (PREDICT): a prospective observational study. Lancet Neurol 2012;11:307-14.

19. Davis SM, Broderick J, Hennerici M, et al. Hematoma growth is a determinant of mortality and poor outcome after intracerebral hemorrhage. Neurology 2006;66:1175-81.

20. Mayer SA. Ultra-early hemostatic therapy for intracerebral hemorrhage. Stroke 2003;34:224-9.

21. Wartenberg KE, Mayer SA. Ultra-Early Hemostatic Therapy for Intracerebral Hemorrhage: Future Directions. Front Neurol Neurosci 2015;37:107-29.

22. Chinese Pharmacopoeia Committee. Chinese pharmacopoeia. Beijing:China medical science and technology press. 2010:111-3.

23. Gz S. preliminary clinical study of herbal medicine on hemorrhagic stroke: Removing Blood Stasis Therapy. Tianjin Medical Journal 1982;08:473-6.

24. The Professional Board of Neurology Department of Chinese Association of Integrative Medicine in Beijing, GAO L. Expert consensus on hypertensive intracerebral hemorrhage in acute stage in diagnosis and treatment combining traditional Chinese medicine and Western medicine [J]. Chinese General Practice 2016;19:3641-8. Chinese.

25. Zou YH, Bin M. Guidelines for the management of spontaneous intracerebral hemorrhage: a guideline for healthcare professionals from the China Association of Chinese Medicine. Chinese traditional Chinese medicine modern remote education 2011:9:110-2. Chinese.

26. Brouwers HB, Falcone GJ, McNamara KA, et al. CTA spot sign predicts hematoma expansion in patients with delayed presentation after intracerebral hemorrhage. Neurocrit Care 2012;17:421-8.

27. Bin L, Jian L. Clinical observation of early use promoting blood circulation and removing blood stasis herbal injection to treat acute intracerebral hemorrhage. Shandong Journal of Traditional Chinese Medicine 2000;19:461-2.

28. Anderson CS, Heeley E, Huang Y, et al. Rapid blood-pressure lowering in patients with acute intracerebral hemorrhage. N Engl $J$ Med 2013;368:2355-65.

29. Lyden PD, Shuaib A, Lees KR, et al. Safety and tolerability of NXY059 for acute intracerebral hemorrhage: the CHANT Trial. Stroke 2007;38-2262-9. 